\title{
Use of ultrasound for the assessment of dehydration in pediatric patients with mild to moderate dehydration
}

\author{
Yalda Ravanshad, ${ }^{1}$ Anoush Azarfar, ${ }^{2}$ Seied Ali Alamdaran, ${ }^{3}$ Mitra Naseri, ${ }^{4}$ \\ Gholamreza Sarvari, ${ }^{4}$ Sepideh Bagheri, ${ }^{4}$ Armin Vahabi Sani ${ }^{3}$ \\ ${ }^{1}$ Department of Community Medicine, Mashhad Branch, Islamic Azad University, Mashhad; \\ ${ }^{2}$ Kidney Transplantation Complications Research Center, Mashhad University of Medical Sciences, \\ Mashhad; ${ }^{3}$ Department of Radiology, Faculty of Medicine, Mashhad University of Medical Sciences, \\ Mashhad; ${ }^{4}$ Dr. Sheikh's Children Hospital, Department of Pediatric Diseases, Faculty of Medicine, \\ Mashhad University of Medical Sciences, Mashhad, Iran
}

\begin{abstract}
Management of children with acute gastroenteritis is based upon dehydration estimation. There is no clinical or paraclinical tool which exactly estimates the dehydration degree. Recently ultrasonographic parameters as inferior vena cava (IVC) diameter and aorta $(\mathrm{AO})$ have been used in some studies for this purpose.
\end{abstract}

\footnotetext{
Correspondence: Sepideh Bagheri, Dr. Sheikh's Children Hospital, Department of Pediatric Diseases, Faculty of Medicine, Mashhad University of Medical Sciences, Mashhad, Iran.

Tel.: +989155255451.

E-mail: Bagheris@mums.ac.ir
}

Key words: Aorta; Dehydration; Gastroenteritis; Inferior vena cava; Pediatric; Ultrasonography.

Acknowledgments: the authors wish to express their special thanks to patients and their families for their kind cooperation and also to Dr. Sheikhs Emergency Department staff for their contribution.

Contributions: YR, AA, conceived and designed the analysis, collected data, drafting of manuscript and final revision; SAA, AVS, acquisition of data, analysis and interpretation of data, critical revision; MN, GS, study concept, interpretation of data, writing manuscript and final revision; $\mathrm{SB}$, study design, data interpretation, drafting of manuscript and final revision.

Conflict of interest: the authors declare no potential conflict of interest.

Funding: none.

Ethical statement: the study was approved by the medical ethics committee of Mashhad University of Medical Sciences and all illegible patients' parents or caregivers signed the informed consent.

Note: this manuscript is derived from MD thesis of Dr. Armin Vahabi Sani (84136).

Received for publication: 5 March 2019

Revision received: 10 June 2019.

Accepted for publication: 1 July 2019.

This work is licensed under a Creative Commons Attribution 4.0 License (by-nc 4.0).

${ }^{\circ}$ Copyright: the Author(s), 2019

Licensee PAGEPress, Italy

Emergency Care Journal 2019; 15:8151

doi:10.4081/ecj.2019.8151
This study aims to evaluate the efficacy of ultrasound in detecting mild and moderate degrees of dehydration in children. The study was performed in the emergency department of Dr. Sheikh's Children Hospital, Mashhad, Iran. Children with mild to moderate degrees of dehydration according to World health Organization (WHO) clinical scale were enrolled. Their inferior vena cava diameters, aorta and IVC/AO ratio were measured before and after fluid therapy using ultrasound. Ultrasound was performed by two pediatric sonographers. 36 patients (mean age of $16.94 \pm 11.02$ months) entered the study. 11 patients had mild and 25 moderate dehydration according to WHO clinical scale. All 11 patients with mild dehydration received oral rehydration. 13 patients in the moderate dehydration group received intravenous rehydration because of oral intolerance to fluids and recurrent vomiting. IVC diameter and IVC/AO ratio after fluid therapy in children with both mild and moderate dehydration degrees was significantly greater $(\mathrm{P}<0.001)$. However, we did not observe any significant difference in aorta diameter before and after fluid therapy. Using Receiver Operating Characteristic (ROC) curve, the proper cut-off point of IVC/AO ratio to differentiate patients with moderate dehydration from mild dehydration is equal to 0.782 with sensitivity and specificity equal to $88 \%$ and $45.45 \%$ respectively. Further, the area under the ROC curve for this cut-off is equal to 0.569 . In conclusion, ultrasonography cannot differentiate between mild and moderate dehydration degrees, but studies with larger population of patients should be performed.

\section{Introduction}

Acute gastroenteritis with dehydration is still one of the most common conditions encountered in the pediatric emergency departments (ED). ${ }^{1}$ Estimating the degree of dehydration and thus choosing the appropriate treatment method is a challenge for physicians working in EDs. Weight loss is a good predictor of dehydration degree and it is considered as the clinical gold standard for dehydration assessment ${ }^{2}$ but, since in most cases recent weights are not available, this method is of limited value. Most physicians estimate the degree of dehydration according to a number of signs and symptoms, ${ }^{3}$ which demonstrated low sensitivity and specificity. ${ }^{4,5}$ Current guidelines correlate the main signs and symptoms to different categories of dehydration severity, with the aim of better stratifying patients and consequently optimizing their clinical and therapeutic management.

Studies on the use of laboratory indices for assessment of dehydration have shown that these parameters like urea or serum bicarbonate are also ineffective in dehydration degree assessment and no definitive laboratory test for the assessment of dehydration 
is actually available., ${ }^{4,6}$ So the need for a rapid and accurate method of dehydration assessment in children seems mandatory. Ultrasonography has been recently used as a tool for dehydration and volume state assessment. ${ }^{7,8}$ The role of point of care ultrasound has been evaluated in several studies for fluid responsiveness identification in the critically ill with the aim of optimizing therapeutic choices: the main ultrasound parameters analyzed in the literature are caval index, ${ }^{9}$ inferior vena cava/aorta (IVC/AO) ratio $^{7,10}$ and IVC diameter respiratory variation. ${ }^{11,12}$ Most studies were performed in populations of critically ill adult patients with hypovolaemic or undifferentiated shock status, in which blind therapeutic choices may result in unfavorable course.

In this study we aimed to evaluate the efficacy of AO/IVC ratio in dehydration assessment in children with mild to moderate clinical dehydration.

\section{Materials and Methods}

A prospective observational study was conducted in the emergency department of Dr. Sheikh children's hospital in Mashhad North of Iran. This hospital is affiliated with the Mashhad University of Medical Sciences. The study was approved by the medical ethics committee of Mashhad University of Medical Sciences and all illegible patients' parents or caregivers signed informed consent.

Children with a diagnosis of gastroenteritis who were mildly or moderately dehydrated according to World Health Organization (WHO) dehydration clinical scale entered the study. Exclusion criteria were as follows: children with severe dehydration, chronic conditions like congenital heart disease or chronic kidney disease, acute blood loss, diuretic therapy, patients with failure to thrive in whom dehydration might be overestimated and patients who presented when our sonographers were not available.

Patient's dehydration degree and weight was assessed on presentation and then a targeted point of care ultrasound was performed by two pediatric sonographers before any treatment. However, we can train pediatricians to perform these sonograms in EDs especially in cases of severe dehydration and shock to guide them in treatment. Sonography probe was placed in the subxiphoid space and maximal diameter of the IVC was measured during expiration, maximum diameter of the Aorta was also measured during systole. Data were recorded and patients received the necessary treatments according to their physician's order who were blinded to the results of the sonography.

IVC and Aorta diameters were again measured after complete rehydration of patients and their weight was also measured and recorded again.

\section{Statistical analysis}

Data were analyzed using SPSS 16 statistical package. Data were presented using descriptive statistics including means, standard deviation and proportions. We used students $\mathrm{T}$ test for quantitative data and chi-square test for qualitative data. KolmogorovSmirnov test was used to test the normality of the independent variables. For all parameters level of significance in terms of Pvalue was 0.05 .

\section{Results}

36 children with gastroenteritis who were mild or moderately dehydrated according to WHO clinical scale were studied. 15 were male and 21 were female. Mean age of patients was $16.94 \pm 11.02$ months (range: 4-54 months).

Mean patients weight before fluid therapy was $10.06 \pm 2.74 \mathrm{~kg}$. After proper oral or intravenous fluid-therapy according to the physicians decision this was $11.24 \pm 3.65 \mathrm{~kg}$. The dehydration degree and the fluid intake volume were documented as $7.94 \pm 2.45$ percent and $418.75 \pm 103.08 \mathrm{~mL}$. Further, the aorta and IVC diameters before the fluid therapy were measured as $7.08 \pm 0.99 \mathrm{~mm}$ and $4.94 \pm 1.36 \mathrm{~mm}$ respectively, and after suitable fluid therapy were measured as $7.09 \pm 0.87 \mathrm{~mm}$ and $6.87 \pm 1.10 \mathrm{~mm}$. The IVC to AO diameter ratio was therefore calculated to be $0.70 \pm 0.16$ and $0.97 \pm 0.07$ respectively before and after fluid therapy. Based on clinical scales, $69.44 \%$ percent of patients (25 patients) had moderate dehydration, and $30.56 \%$ (11 patients) had mild dehydration.

Analyzing the correlation of patients age, aorta and IVC diameters and their ratio we observed that in children with mild to moderate dehydration, there is a significant difference between aorta and IVC diameters and the age of patients, both before and after fluid therapy. However, we did not observe any significant difference between IVC/AO ratio and the age of patients in any group. Comparing the aorta diameter before and after fluid therapy in patients with mild dehydration, moderate dehydration and overall, using Wilcoxon paired t-test shows that aorta diameter is not significantly changed after fluid therapy (Table 1).

Applying independent t-test to IVC/AO diameter ratio in both groups of mild $(\leq 5 \%)$ and moderate dehydration $(>5 \%)$, we observed that the mean IVC/AO diameter ratio shows no significant differences at the level of $5 \%$ in both groups (Table 2).

To find the best cut-off point for IVC/AO diameter ratio to distinguish mild and moderate dehydration levels, ROC curve analysis was used. As shown in Figure 1, the best cut-off point was found to be 0.782 with sensitivity and specificity of $88 \%$ and $45.45 \%$ respectively. The area under the ROC curve (AUC) for this point is equal to 0.569 (Figure 1).

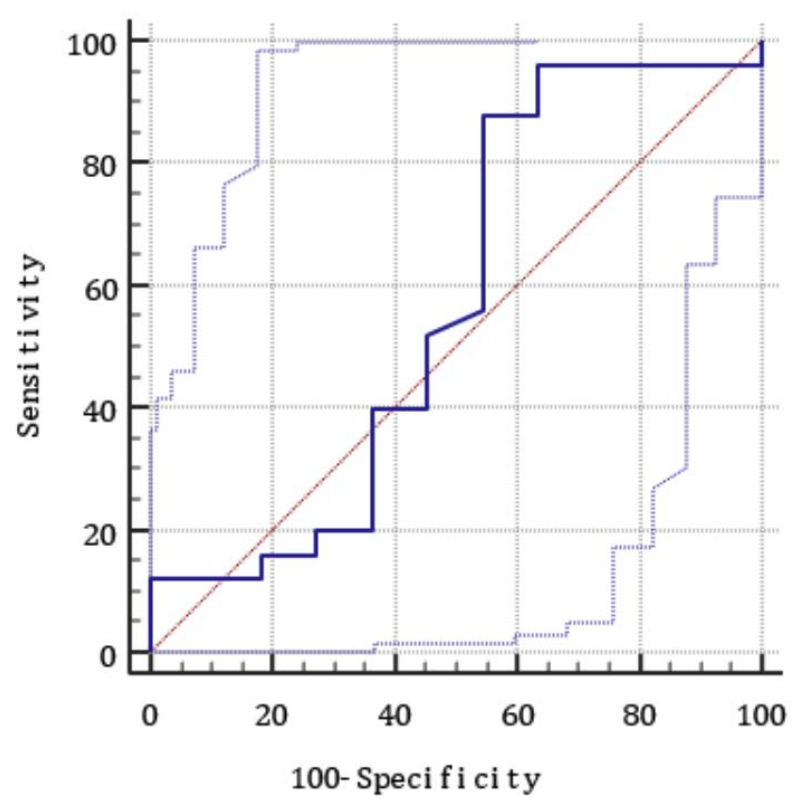

Figure 1. Receiver Operating Characteristic curve for inferior vena cava/aorta diameter ratio. 


\section{Discussion}

Estimating the dehydration degree is the most important aspect of gastroenteritis management in pediatric patients. ${ }^{1}$ Objective dehydration assessments which are frequently used are both inaccurate and difficult. Studies using ultrasound measurements of IVC and aorta have been conducted in pediatric population for dehydration degree assessment. ${ }^{7,10}$

These studies have mostly evaluated children with severe dehydration. We conducted this study to evaluate if ultrasonographic findings can help in distinguishing mild and moderate degrees of dehydration.

In 1979, Natori et al. reported a correlation between the variation of the IVC diameter and the right ventricular blood pressure. ${ }^{13}$ However, the inherent limitations of ultrasound, such as lack of skilled personnel and reliable reference values for the IVC diameter in children and adults, bounded the use of ultrasound for this purpose. ${ }^{13,15}$

Corl et al., in a study on critical ill patients found that point of care ultrasound and IVC diameter measurement can detect fluid responsiveness and can be used to guide fluid resuscitation in the EDs. ${ }^{16}$

Results of the current study show that, IVC and Aorta diameters are not fully appropriate parameters in mild and moderate dehydration degrees. Kariman et $a l .{ }^{17}$ discussed that the IVC diameter, in Sagittal $(\mathrm{P}=0.004)$ and transverse view $(\mathrm{P}=0.001)$, is a proper index to diagnose moderate dehydration. In our study, as well, the IVC diameter after fluid therapy was significantly greater in patients with both mild and moderate dehydration degrees. Moreover, we also observed that the IVC/AO diameter ratio was significantly greater after fluid therapy in both groups $(\mathrm{P}<0.001)$.
Another study by Rahman et al. ${ }^{18}$ reports that IVC diameters, abdominal aorta and IVC/AO diameter ratio significantly changed after therapy. Even though their findings about IVC diameter and IVC/AO diameter ratio were similar to ours, we did not observe any significant difference in aorta diameter before and after fluid therapy. Based on ROC curve, we found that the appropriate cutoff for IVC/AO diameter to differentiate patients with mild and moderate dehydration is equal to 0.782 . This point has a sensitivity and specificity equal to $88 \%$ and $45.45 \%$ respectively. The area under the ROC curve (AUC) for this cut-off point is equal to 0.569 .

In a similar study by Chen et al., ${ }^{19}$ it was reported the cut-off point of 0.8 for IVC/AO diameter ratio, with sensitivity and specificity equal to $86 \%$ and $56 \%$ respectively, to distinguish patients with severe dehydration $(\geq 5 \%)$. AUC was equal to 0.73 for that cut-off point.

Levine $e t a l .{ }^{10}$ reported the best cut-off point for IVC/AO ratio equal to 1.22 with sensitivity and specificity equal to $93 \%$ and $59 \%$ respectively, while the best cut-off point based on WHO standards shows $73 \%$ sensitivity and $43 \%$ specificity. In the study by $\mathrm{Ng}$ et $a l .,{ }^{20}$ no significant relation between IVC/AO diameter ratio and circulating blood volume was observed.

These different findings may be due to differences in estimating dehydration degrees in patients since no clinical scale can estimate dehydration degree quite accurately. On the other hands, parameters used to identify the cut off points were not the same in these studies.

Results of this study might have been affected by its relatively small sample: studies with larger populations may show better results and can be better used to assess ultrasound accuracy for dehydration estimation. Sonographers skills might also play an important role in the accuracy of this method.

Table 1. Inferior vena cava/aorta diameter before and after fluid therapy.

\begin{tabular}{|c|c|c|c|c|c|}
\hline Dependent variable & Group & Variable & Mean & Standard deviation & Significance level \\
\hline \multirow[t]{5}{*}{ AO diameter } & Mild dehydration & $\begin{array}{l}\text { Before fluid therapy } \\
\text { After fluid therany }\end{array}$ & 7.38 & 0.97 & NS \\
\hline & Moderate dehydration & Before fluid therapy & 6.99 & 0.98 & NS \\
\hline & & After fluid therapy & 6.97 & 0.82 & \\
\hline & Mixed (any level of dehydration) & Before fluid therapy & 7.11 & 0.98 & NS \\
\hline & & After fluid therapy & 7.09 & 0.87 & \\
\hline \multirow[t]{6}{*}{ IVC diameter } & Mild dehydration & Before fluid therapy & 5.52 & 1.81 & $\mathrm{P}<0.001$ \\
\hline & & After fluid therapy & 7.13 & 1.33 & \\
\hline & Moderate dehydration & Before fluid therapy & 4.74 & 1.04 & $\mathrm{P}<0.001$ \\
\hline & & After fluid therapy & 6.75 & 0.99 & \\
\hline & Mixed (any level of dehydration) & Before fluid therapy & 4.99 & 1.35 & $\mathrm{P}<0.001$ \\
\hline & & After fluid therapy & 6.87 & 1.1 & \\
\hline \multirow[t]{6}{*}{ IVC/AO diameter ratio } & Mild dehydration & Before fluid therapy & 0.74 & 0.18 & $\mathrm{P}<0.001$ \\
\hline & & After fluid therapy & 0.97 & 0.72 & \\
\hline & Moderate dehydration & Before fluid therapy & 0.68 & 0.15 & $\mathrm{P}<0.001$ \\
\hline & & After fluid therapy & 0.97 & 0.08 & \\
\hline & Mixed (any level of dehydration) & Before fluid therapy & 0.7 & 0.15 & $\mathrm{P}<0.001$ \\
\hline & & After fluid therapy & 0.96 & 0.07 & \\
\hline
\end{tabular}

$\mathrm{AO}$, aorta; IVC, inferior vena cava; NS, not significant.

Table 2. Inferior vena cava/aorta diameter comparison in two groups of mild and moderate dehydration.

\begin{tabular}{lccc} 
Dehydration degree & No. patients & Mean & Standard deviation \\
Mild dehydration & 11 & 0.74 & 0.18 \\
Moderate dehydration & 25 & 0.68 & 0.321 \\
\hline
\end{tabular}




\section{Conclusions}

Results of the present study show that ultrasonographic estimation of IVC/Ao ratio cannot differentiate between mild and moderate degrees of dehydration. However, this IVC/Ao ratio and IVC diameter resulted significantly greater after appropriate fluid therapy in patients with both mild and moderate degrees of dehydration. The best cut-off point for IVC/Ao ratio was 0.782 which is associated with a high sensitivity but low specificity for diagnosis of moderate dehydration.

\section{References}

1. Azarfar A, Ravanshad Y, Keikhosravi A, et al. Rapid rehydration to correct dehydration and resolve vomiting in children with acute gastroenteritis. Turk J Emerg Med 2014;14:111-4.

2. Friedman JN, Goldman RD, Srivastava R, Parkin PC. Development of a clinical dehydration scale for use in children between 1 and 36 months of age. J Pediatr 2004;145:201-7.

3. Steiner MJ, DeWalt DA, Byerley JS. Is this child dehydrated? JAMA 2004;291:2746-54.

4. Gorelick MH, Shaw KN, Murphy KO. Validity and reliability of clinical signs in the diagnosis of dehydration in children. Pediatrics 1997;99:e6.

5. Rudolph CR, Hostetter M, Lister G, Siegel N. Rudolph's Pediatrics. 21st ed. New York, NY: McGraw-Hill; 2003.

6. Yilmaz K, Karabokuoglu M, Citak A, Uzel N. Evaluation of laboratory tests in dehydrated children with acute gastroenteritis. J Pediatr Child Health 2002;38:226-8.

7. Chen L, Kim Y, Santucci KA. Use of ultrasound measurement of the inferior vena cava diameter as an objective tool in the assessment of children with clinical dehydration. Acad Emerg Med 2007;14:841-5.

8. Boyd JH, Sirounis D. Assessment of adequacy of volume resuscitation. Curr Opin Crit Care 2016;22:424-7.

9. Pershad J, Myers S, Plouman C, et al. Bedside limited echocardiography by the emergency physician is accurate during evaluation of the critically ill patient. Pediatrics 2004;114:e667.

10. Levine AC, Shah SP, Umulisa I, et al. Ultrasound assessment of severe dehydration in children with diarrhea and vomiting.
Acad Emerg Med 2010;17:1035-41.

11. Zhang Z, Xiao X, Sheng Y, Lei X. Ultrasonographic measurement of the respiratory variation in the inferior vena cava diameters in predictive of fluid responsiveness in critically ill patients: Systematic review and meta-analysis. Ultrasound Med Biol 2014;40:845-53.

12. Long E, Duke T, Oakley E, et al. Does respiratory variation of inferior vena cava diameter predict fluid responsiveness in spontaneously ventilating children with sepsis. Emerg Med Australas 2018;30:556-63.

13. Natori H, Tamaki S, Kira S. Ultrasonographic evaluation of ventilatory effect on inferior vena caval configuration. Am Rev Respir Dis 1979;120:421-7.

14. Rosenthal D, Wellons ED, Levitt AB, et al. Role of prophylactic temporary inferior vena cava filters placed at the ICU bedside under intravascular ultrasound guidance in patients with multiple trauma. J Vasc Surg 2004;40:958-64.

15. Kosiak W, Swieton D, Piskunowicz M. Sonographic inferior vena cava/aorta diameter index, a new approach to the body fluid status assessment in children and young adults in emergency ultrasound - preliminary study. Am J Emerg Med 2008;26:320-5.

16. Corl KA, George NR, Romanoff AJ, et al. Inferior vena cava collapsibility detects fluid responsiveness among spontaneously breathing critically-ill patients. J Crit Care 2017;41:130-7.

17. Kariman H, Heidarian A, Majidi A, et al. Accuracy of inferior vena cava, aorta, and jugular vein ultrasonographic diameters in identifying pediatric dehydration. Iran $\mathrm{J}$ Emerg Med 2015;2:174-81.

18. Rahman NH, Ahmad R, Kareem MM, Mohammed MI. Ultrasonographic assessment of inferior vena cava/abdominal aorta diameter index: a new approach of assessing hypovolemic shock class 1. Int J Emerg Med 2016;9:8.

19. Chen L, Hsiao A, Langhan M, et al. Use of bedside ultrasound to assess degree of dehydration in children with gastroenteritis. Acad Emerg Med 2010;17:1042-7.

20. Ng L, Khine H, Taragin BH, et al. Does bedside sonographic measurement of the inferior vena cava diameter correlate with central venous pressure in the assessment of intravascular volume in children? Pediatr Emerg Care 2013;29:337-41. 